\title{
Digital Filter Approach for Simulation of a Complex Integrated Laser Diode Based on the Traveling-Wave Model
}

\author{
Wei Li, Member, IEEE, Wei-Ping Huang, Senior Member, IEEE, and Xun Li, Member, IEEE
}

\begin{abstract}
The traveling-wave model is commonly used in the simulation of semiconductor laser diodes and photonic integrated circuits. When the laser diode is cascaded with other passive elements of relatively large optical dimension or complicated structure such as a sophisticated distributed Bragg reflector section, the conventional approach by tracing the forward and the backward traveling waveform along the entire passive section in the time domain becomes time consuming or technically impossible. To overcome this difficulty, a split-step approach is proposed in this study. Since the spectral characteristics of the passive sections are known from analytical/numerical calculations or experimental measurement, the effective time-domain digital filters are introduced for the passive components. Therefore, in the simulation, the laser diode part is still modeled by the traveling-wave approach, but the digital filters are used to model the passive components butted at the end of the active part, which interact with the optical field of the laser diode at the interface.
\end{abstract}

Index Terms-Digital filters, frequency-domain analysis, laser diodes, modeling, simulation, time-domain analysis.

\section{INTRODUCTION}

$\mathbf{S}$ EMICONDUCTOR laser diodes are one of the key components in a variety of applications such as optical communications and signal processing. As fabrication technology becomes more mature, complex integrated configurations of laser cavities can be realized to achieve different performance specifications. In the investigation of this kind of photonics circuit, numerical simulation and modeling is playing a more and more important role. In the modeling and simulation of laser diodes, there have been two different formalisms, namely, the standing-wave approach [1]-[4] and the traveling-wave approach [2], [5]-[7]. The standing-wave approach is based on the assumption that the temporal and the spatial dependence of field distributions of the cavity modes are separable. As such, the dynamics is considered in the modal amplitudes. Consequently, the standing-wave approach is valid only when the photon lifetime is much shorter than the characteristic time of the laser dynamics. The traveling-wave model, on the other hand, makes no assumptions about the cavity modes. Rather, it solves the time-dependent coupled-wave equations for the forward the backward traveling waves directly and therefore is valid even when the laser cavity has a relatively small $Q$-factor

Manuscript received October 7, 2003; revised December 23, 2003

W. Li is with the Department of Chemistry and Engineering Physics, University of Wisconsin, Platteville, WI 53818-3099 USA.

W.-P. Huang and X. Li are with the Department of Electrical and Computer Engineering, McMaster University, Hamilton, ON L8S 4L8, Canada.

Digital Object Identifier 10.1109/JQE.2004.826436 and/or the characteristic time of the laser dynamics is very short. Another advantage of the traveling-wave model is that it can be readily applied to laser diodes operated with multiple cavity modes, for which the standing-wave model may have difficulty in finding the complex roots corresponding to each mode. Therefore, it has broad applications on the integrated optical devices and subsystems recently [8]-[10].

However, to extend the application of the traveling-wave model to the case of complicated photonics circuit which consists of both active and sophisticated passive parts is not straightforward. Zhai et al. have used the novel scattering matrix method in combination with transmission line model of laser to investigate the mode-locking laser with fiber Bragg grating [11]. It is also reported that, in the pioneer VPI products, it is allowed for the digital filters based on transfer-matrix analysis of Bragg gratings to be combined with the time-domain model of laser diodes [8]. One of the challenging tasks for the traveling-wave model is related to tracing the waveform in both the time and spatial domains with the signal transmission speed constrain $\Delta z=v_{g} \Delta t$, where $\Delta z$, and $\Delta t$ are the discretization elements in the spatial and the time domains, respectively, and $v_{g}$ is the group velocity. For instance, it would be time consuming to simulate a laser diode cascaded with a long distributed Bragg reflector (DBR) section (e.g., co-directional coupling filter) using the full traveling-wave approach. Furthermore, under certain circumstances, the passive section may be characterized in terms of measured spectral responses or be calculated analytically/numerically far different from the traveling-wave approach, which cannot be simulated by transfer matrix method, for example, an arrayed waveguide grating (AWG) device, and therefore difficult to be modeled in a self-consistent traveling-wave form as for the laser diode. To overcome the above shortcomings, we will investigate the digital filter approach for the integration of the laser diode with complicated passive components. It should be noted that, due to the circuit nature of the transmission-line model for laser diodes, Lowery already pointed out that the distributed feedback (DFB)/DBR model is similar to a lattice filter [7]. In this study, the digital filter is not referred to our active part modeling. The active part is still simulated by the conventional traveling model or, equivalently, the transmission-line model [7], [9], but the whole passive part is represented by the digital filters. By modeling the passive components along the optical path as two digital filters butted at the end of the active part, one for the reflection at the junction and the other for the transmission at the other end of the passive part, we can 
simulate the counterpropagating waves by a combination of the standard evolution scheme (as done in the full traveling-wave model) and the convolution scheme commonly used in signal processing [14]. It is demonstrated that the new method is more efficient than the full traveling-wave model. More importantly, we can readily incorporate the spectral properties of the passive sections, which can be obtained from measurements or other analytical/numerical models such as the beam propagation method (BPM). Our method is different from [11]. It is similar to the approach of [7], but we will present a general recipe suitable for all complicated integrated devices.

In Section II, we present the theory in details. In Section III, the validity of the method is confirmed and some applications are demonstrated. Finally, we give a brief conclusion.

\section{THEORETICAL FORMULATIONS}

\section{A. Traveling-Wave Model for the Active Sections}

For the active sections, we apply the full traveling-wave models. The governing equations for the envelopes $F(z, t)$ and $R(z, t)$ of the forward and backward traveling optical fields are

$$
\begin{aligned}
& \frac{1}{v_{g}} \frac{\partial F}{\partial t}+\frac{\partial F}{\partial z}=\left(\Gamma g-a_{s}-j \delta\right) F+j \kappa_{F} R+s_{F} \\
& \frac{1}{v_{g}} \frac{\partial R}{\partial t}+\frac{\partial R}{\partial z}=\left(\Gamma g-a_{s}-j \delta\right) R+j \kappa_{R} F+s_{R}
\end{aligned}
$$

where $v_{g}$ is the group velocity, $\Gamma$ is the optical confinement factor, $g$ is the optical gain, $a_{s}$ is the optical loss, and $\delta$ is the detuning factor, respectively. The coupling coefficients due to index/gain/loss gratings are $k_{F}$ and $k_{R}$, which may be complex. The terms $s_{F}$ and $s_{R}$ denote the spontaneous noises associated with the forward and backward traveling waves, respectively. For the sake of simplicity, we assume that the noise terms may be approximated by a complex Gaussian noise with average equal to zero and satisfying the correlation

$$
\begin{aligned}
\left\langle s_{F, R}(z, t) s_{F, R}{ }^{*}\left(z^{\prime}, t^{\prime}\right)\right\rangle & =\beta K\left(\frac{\mathrm{BN}^{2}}{L}\right) V_{g} \delta\left(z-z^{\prime}\right) \delta\left(t-t^{\prime}\right) \\
\left\langle s_{F, R}(z, t) s_{F, R}\left(z^{\prime}, t^{\prime}\right)\right\rangle & =0
\end{aligned}
$$

where $L$ is the laser cavity length, $\beta$ is the spontaneous coupling factor, and $K$ is Petermann's coefficient.

On the other hand, the average carrier density in the active region along the laser cavity is described by the carrier rate equation

$$
\frac{d N}{d t}=\frac{I}{e V}-\frac{N}{\tau}-\mathrm{BN}^{2}-\mathrm{CN}^{3}-2 g v_{g} P
$$

where $N(z, t)$ is the average carrier density, $I(z, t)$ is the injected current, and $V$ is the active region volume of the laser diode. The constant $\mathrm{e}$ is the free electron charge. The parameter $\tau$ stands for the electron lifetime and $B, C$ are bimolecular and Auger recombination coefficients, respectively. $P$ is the photon density, which is related to the magnitude of the traveling-wave fields as

$$
P=|F|^{2}+|R|^{2}
$$

The optical gain is modeled by

$$
g=\frac{g_{N}\left(N-N_{T}\right)}{2(1+\varepsilon P)}
$$

where $g_{N}$ is the differential gain, $N_{T}$ is the transparent carrier density, and $\varepsilon$ is the gain compression factor. The effective refractive index can be expressed as

$$
n_{\mathrm{eff}}=n_{\mathrm{eff} 0}-\Gamma a_{m} g \frac{\lambda_{0}}{4 \pi}
$$

where $n_{\text {effo }}$ is the intrinsic refractive index, $a_{m}$ is the linewidth enhancement factor, and $\lambda_{0}$ is the reference wavelength which is chosen closed to the lasing wavelength. The detuning factor in (1) is defined as

$$
\delta=\frac{2 \pi}{\lambda_{0}} n_{\mathrm{eff}}-\frac{\pi}{\Lambda}
$$

where $\Lambda$ is the grating period of the DFB lasers. For the FabryPérot laser case, we only need to set the coupling coefficient $k$ equal to zero, and replace $\pi / \Lambda$ by $(2 \pi / \Lambda) n_{\text {effo }}$ and assume that the reference wavelength is the gain peak wavelength. The finite difference scheme of the above equations is standard, which can be found in our previous work [9] or some recent works [10], [11], but the optical field at the end of the laser cavity should be calculated using the new scheme described in the following section, i.e., the end field will interact with the reflection digital filter extracted from the whole passive component.

Digital Filter for Passive Section: For simplicity, we use a grating formed passive section as an example. In the absence of nonlinearity, the governing equations for the envelopes $F(z, t)$ and $R(z, t)$ of the forward and backward traveling optical fields are

$$
\begin{aligned}
& \frac{1}{v_{g}} \frac{\partial F}{\partial t}+\frac{\partial F}{\partial z}=\left(\alpha-j \delta_{o}\right) F+j \kappa_{F} R \\
& \frac{1}{v_{g}} \frac{\partial R}{\partial t}-\frac{\partial R}{\partial z}=\left(\alpha-j \delta_{o}\right) R+j \kappa_{R} F .
\end{aligned}
$$

As the governing equations are linear, we may perform a Fourier transform so that we have

$$
\begin{aligned}
\frac{\partial \widetilde{F}}{\partial z} & =(\alpha-j \delta) \widetilde{F}+j \kappa_{F} \widetilde{R} \\
-\frac{\partial \hat{R}}{\partial z} & =(\alpha-j \delta) \widetilde{R}+j \kappa_{R} \widetilde{F} .
\end{aligned}
$$

Equation (9) can be readily solved by using the transfer matrix method for arbitrary $\alpha, \delta$, and $k$ and the characteristic matrix of the passive section can then be found by the multiplication of the transmission matrix of each subsection along the waveguide axis. We may further convert the characteristic transfer matrix into the scattering matrix with the reflection coefficient and the transmission coefficient from the passive section given by a complex function $\widetilde{R}(f)$ and $\widetilde{T}(f)$, respectively.

In the digital filter implementation, the so-called causal or physically realizable filter is used, which provides the output at a specific time based on the inputs at that specific time or earlier [14], [15]. There are two commonly used linear filters in this category. One is a recursive filter (IIR); the other is nonrecursive (FIR). It is argued that, for the same number of coefficients, the recursive filter is usually more accurate than the nonrecursive one [15]. However, if coefficients are not chosen well, the recursive approach may cause unstable solutions. Since in our applications the number of coefficient is not of great concern, we adapt the nonrecursive filter in the simulation.

As shown in Fig. 1, the output optical field $F_{\text {out }}^{n}$ at a specific time $n$ can be expressed by the $M$ input optical field $F_{\text {end }}^{k}$ at 


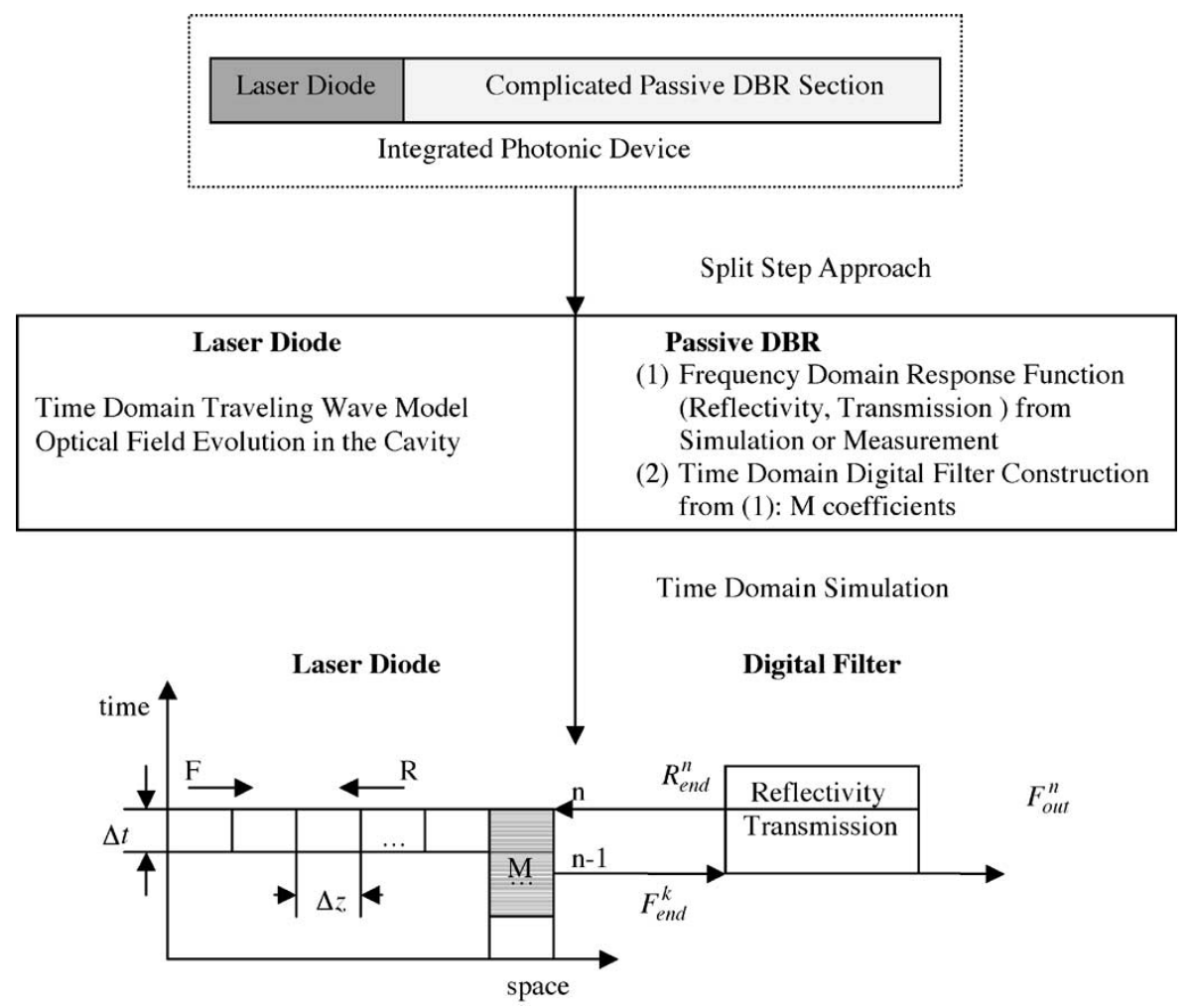

Fig. 1. Schematic of the digital filter approach for the traveling-wave model of the laser diode.

this specific time and earlier at the interface of the active and passive parts as

$$
F_{\text {out }}^{n}=\sum_{k=0}^{M} T^{k} F_{\text {end }}^{n-k}
$$

where $T^{k}$ is the digital filter coefficients, which can be determined from the response function, i.e., the transmission coefficient of the passive components in the frequency domain

$$
\widetilde{T}(f)=\sum_{k=0}^{M} T^{k} \exp (-2 \pi i k f \Delta t)
$$

From (11), we find that $T^{k}$ could be obtained easily by a reverse fast Fourier transform (FFT) transformation of the transmission coefficient function in the frequency domain. Similarly, the reflected field $R_{\text {end }}^{n}$ from the passive part to the active part at a specific time $n$ can also be expressed by the $M$ input fields $F_{\text {end }}^{k}$ at this specific time and earlier as

$$
R_{\text {end }}^{n}=\sum_{k=0}^{M} R^{k} F_{\text {end }}^{n-k}
$$

where $R^{k}$ is the digital filter coefficients, which can be determined from the reflectivity coefficient of the passive part in the frequency domain

$$
\widetilde{R}(f)=\sum_{k=0}^{M} R^{k} \exp (-2 \pi i k f \Delta t) .
$$

From (11) and (13), we can see that the extracted filters for the passive components are a set of time sequence complex numbers: $M+1$ for $T^{k}, k=0,1, \ldots, M$, the transmission filter; $M+1$ for $R^{k}, k=0,1, \ldots, M$, the reflection filter.
As we know, the passive component is not always as simple as expressed in (8) and (9), which correspond to the cases of a Bragg grating fiber or DBR. However, the procedure described above is quite general. We may apply this method to any complicated case provided that the effective reflectivity and transmission coefficient are given. Therefore, if a laser diode is cascaded with a long passive section, we can first calculate analytically using the transfer matrix approach or numerically using the BPM approach or even use the measurement data to get the reflectivity and transmission spectrum of the passive section in the frequency domain, and then use (10)-(13) to obtain the reflectivity and transmission filters' coefficients. Instead of tracing the wave along the passive section, these two effective digital filters will be mounted at the end of the laser diode to simulate the whole passive section. The algorithm is shown schematically in Fig. 1. The optical field of the active part of the device, i.e., the laser diode, will evolve explicitly as in the case of the traveling-wave model or transmission-line model, but the feedback field $R_{\text {end }}^{n}$ from the passive components at time $n$ will be determined from the previous $M \times \Delta t$ time step output field $F_{\text {end }}^{k}$, $k=n, n-1, \ldots, n-M$, propagating into the passive section by the reflection digital filter. The laser will "feel" a weighted time delay which in the frequency domain is exactly the response function of the passive section. It is noted that: 1) in the simulation, $\Delta t$ should be small enough to make the Nyquist critical frequency range cover the passive section bandwidth [14], [15] and 2) the digital filter coefficient number $M$ should be chosen such that $M \times \Delta t$ is comparable with the time delay; otherwise, the time delay in the passive waveguide should be considered. Generally to obtain an accurate digital filter for the fixed $M$ is a challenging work in signal processing while $M$ is small, but our scheme is suitable to the long complicated passive 


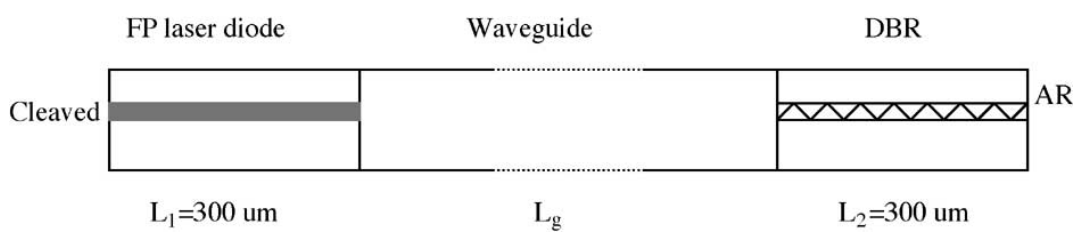

Fig. 2. Device used to perform the comparison between the digital filter and full traveling-wave approaches. Lg is changed from 0 to $1000 \mu \mathrm{m}$.

TABLE I

PARAMETERS USED IN THE SimUlationS

\begin{tabular}{l|l}
\hline Parameters & Values \\
\hline Active layer thickness d $(\mu \mathrm{m})$ & 0.15 \\
Active layer width $\mathrm{w}(\mu \mathrm{m})$ & 3.0 \\
Carrier life time $\tau(\mathrm{ns})$ & 10.0 \\
Bimolecular coefficient $\mathrm{B}\left(10^{-10} \mathrm{~cm}^{3} / \mathrm{s}\right)$ & 1.0 \\
Auger coefficient $\mathrm{C}\left(10^{-27} \mathrm{~cm}^{6} / \mathrm{s}\right)$ & 3.0 \\
Differential gain $\mathrm{g}_{\mathrm{N}}\left(10^{-16} \mathrm{~cm}^{2}\right)$ & 4.0 \\
Absorption and scattering loss $\mathrm{a}_{\mathrm{S}}\left(\mathrm{cm}^{-1}\right)$ & 20.0 \\
Effective index $\mathrm{n}_{\text {effo }}$ & 3.20 \\
Effective group index $\mathrm{n}_{\mathrm{g}}$ & 3.70 \\
Transparent carrier density $\mathrm{N}_{\mathrm{T}}\left(10^{18} \mathrm{~cm}^{-3}\right)$ & 1.0 \\
Line-width enhancement factor $\mathrm{a}_{\mathrm{m}}$ & 4.0 \\
Confinement factor $\Gamma$ & 0.30 \\
Nonlinear gain coefficient $\varepsilon\left(10^{-16} \mathrm{~cm}^{3}\right)$ & 1.0 \\
Spontaneous coupling coefficient $\beta$ & $5 \times 10^{-5}$ \\
Petermann factor K & 1.0 \\
\hline
\end{tabular}

component. $M$ is always very large due to the long time delay as shown in the following examples. Since a nonrecursive (FIR) filter is a Fourier transformation for the frequency-domain response function of the passive components, with so many points, the passive components' information can be expressed in the time-domain filter easily as shown in the following examples. It will be very difficult if the time delay is short, i.e., $M$ is small. We suggest that, in that case, the full traveling-wave and scattering matrix method should be used as it is not necessary to apply the digital filter approach.

\section{SimUlation ResUlTS AND APPLICATIONS}

\section{A. Comparison Between the Two Methods}

To show the validity of the method presented in the previous section, we will peform a series tests using both the full traveling-wave and digital filter approaches as shown in Fig. 2. We have is a $300-\mu \mathrm{m}-$ long FP laser diode and, at the right end, a DBR with $\mathrm{KL}=1$, Bragg wavelength at $1.55 \mu \mathrm{m}$, and the cavity length equal to $300 \mu \mathrm{m}$. Between the laser diode and the $\mathrm{DBR}$, a uniform waveguide connects the two pieces together. We vary the length of waveguide from 0 to $1000 \mu \mathrm{m}$ and compare the output power, carrier/photon distribution within the laser cavity, and output spectrum obtained by the two methods. The active part simulation parameters are listed in Table I. These parameters will be used in the other calculations of this work unless otherwise specified. This imaginary device of course is impractical, but it is quite meaningful when testing the algorithm. Figs. 3-6 show the typical results, and we can see that the digital filter approach is in excellent agreement with the full traveling-wave model. However, while considering the simulation time between the two methods, the digital filter approach will show great advantage over the full traveling-wave model, which has to trace the waveform along the entire passive section. Fig. 7 shows the comparison of the simulation time between the two

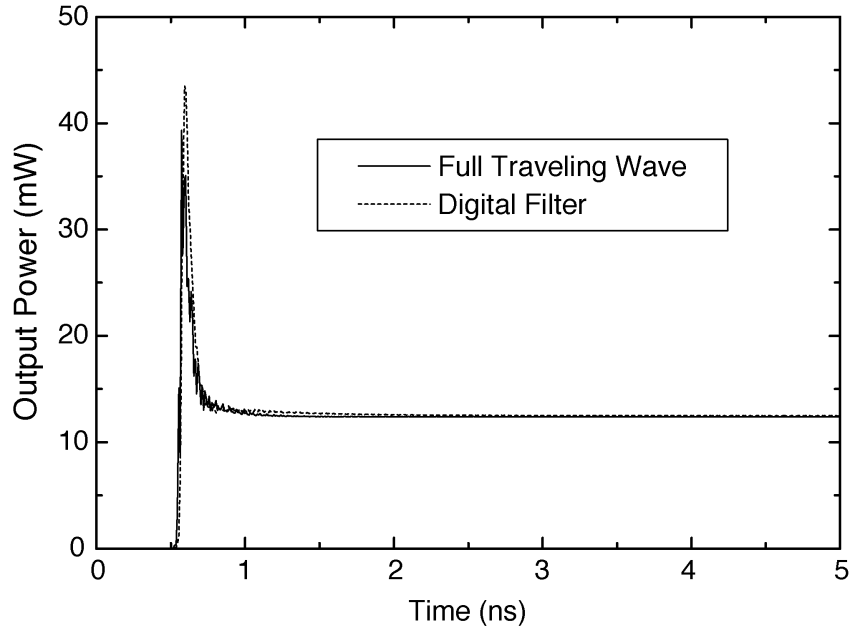

Fig. 3. Output power versus time comparison between the two methods. Here $\mathrm{Lg}=250 \mu \mathrm{m}$. For other cases, similar results are obtained.

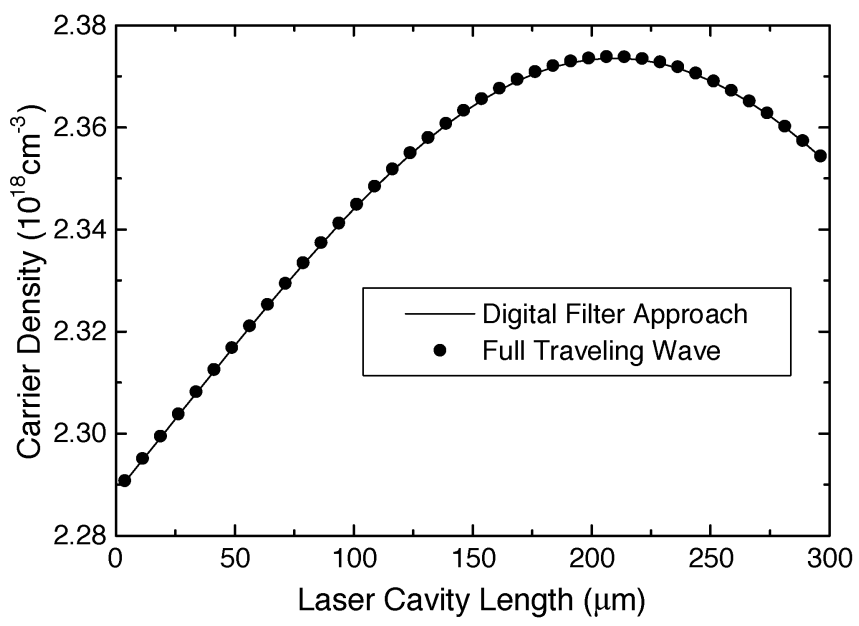

Fig. 4. Carrier distribution comparison between the two methods.

approaches. The proposed digital filter approach simulates all of the complicated passive components by just two filters, one for the reflection and the other for the transmission. In every time step, we only need $M$ (time delay is $M \Delta t$ ) times multiplication operation. However, the conventional method requires a multiple scattering matrix calculation in each finite difference element in the passive part. The longer the passive part is, the more time will be needed. As we know, the undersampling of the conventional method usually makes the simulation very fast in many situations. It will be shown that, for the sample grating case, undersampling may be difficult to implement, Therefore, we conclude that the digital filter approach provide an accurate yet efficient alternative method in the passive section simulation for the traveling-wave model of the laser diode. Most importantly, as we will demonstrate in the following sections, the digital filter approach can also simulate some complicated integrated circuit 


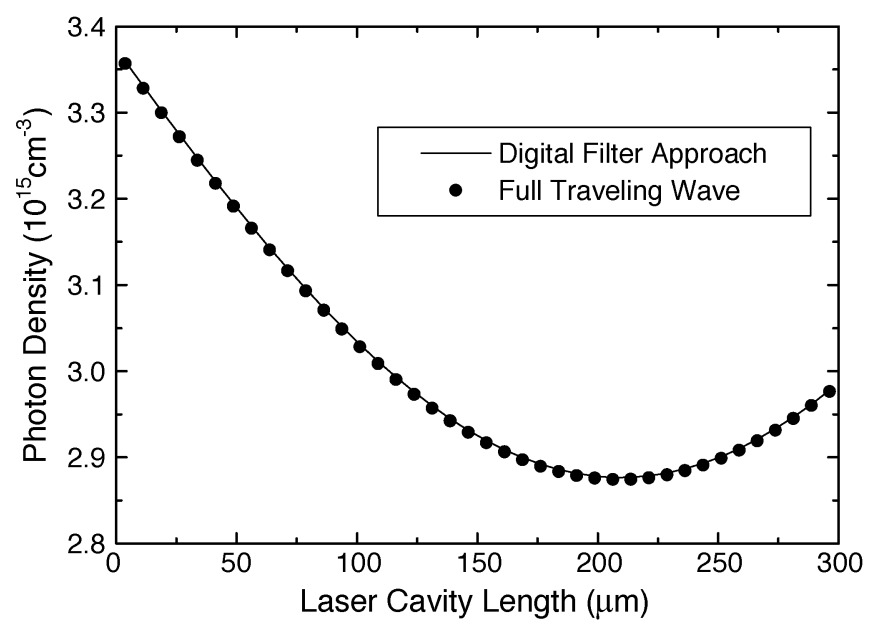

Fig. 5. Photon distribution comparison between the two methods.

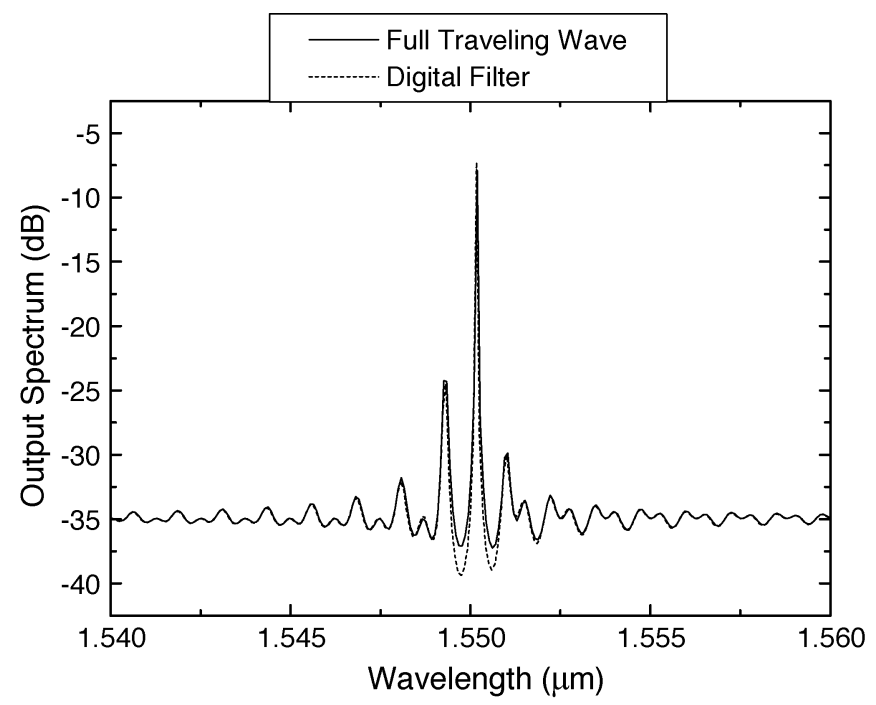

Fig. 6. Output spectra comparison between the two methods. Here $\mathrm{Lg}=$ $0 \mu \mathrm{m}$. For other cases, similar results are obtained except that there are more cavity modes.

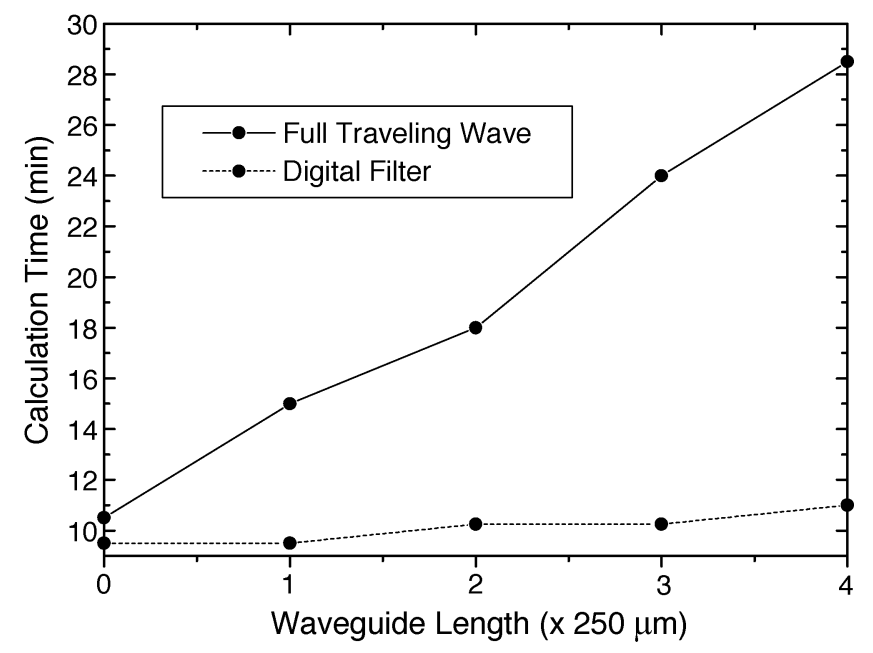

Fig. 7. Comparison of the CPU time used.

for which a full traveling-wave model fails to be applicable. It provides a general recipe to treat the complicated passive/active integrated device. We believe that it is the main merit of the method.

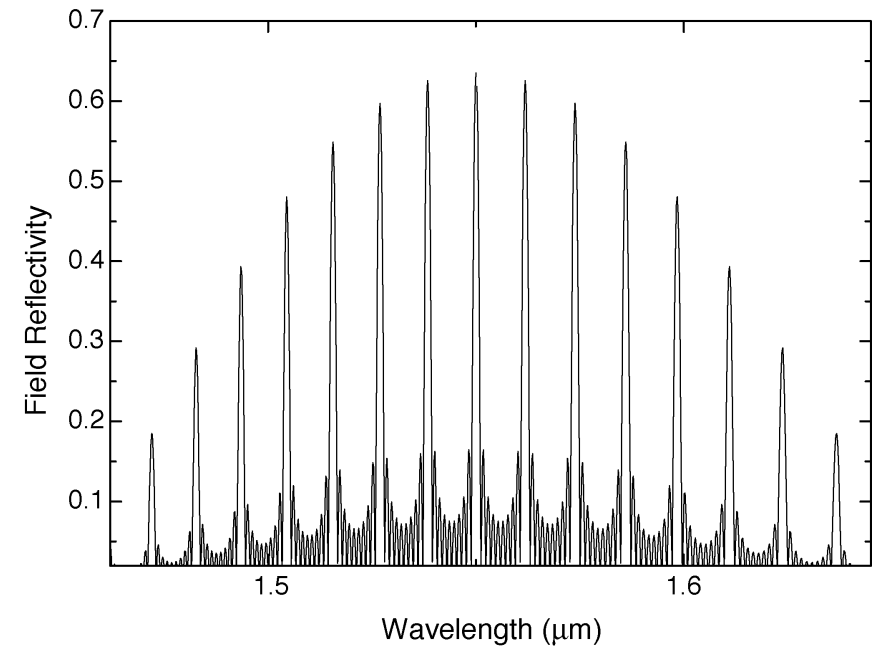

Fig. 8. Typical reflectivity spectrum for the sampled grating waveguide.

\section{B. Sampled Grating DFB Laser Cascaded With a Tunable Filter}

As we know, the sampled grating waveguide can provide a multipeak comb like the reflectivity profile shown in Fig. 8 [16]. Therefore, if this structure is applied in the active section as a sampled grating DFB laser, naturally we should expect a multimode laser diode. It is well known that a uniform grating DFB laser will present two degenerated modes at the edge of the stop band [17]. Therefore, for a sampled grating DFB structure, we should have a multipeak output spectrum, and each peak should consist of two degenerated modes. To make one peak correspond to only one mode, we suggest that a gain coupling should be introduced [9], [18]. To show the above argument clearly, in Fig. 9, we show a typical below-threshold spectrum for a sampled grating DFB laser diode with $10 \%$ gain coupling, which is calculated by the transfer matrix method [19]. It can be seen that this is a potentially multimode laser diode with equal wavelength spacing $\Delta \lambda=\lambda^{2} /\left(2 n_{\text {eff }} \Lambda\right)$. If we can find a filter mirror whose passband is less than the mode interval and also tunable in a wide wavelength range, a wavelength tunable laser with large sidemode suppression ratio (SMSR) will be obtained. A co-directional filter is a good choice to meet this large tuning range requirement [20]-[22].

1) Co-directional Filter: The co-directional filter to be discussed is shown schematically in Fig. 10. A parameter $\gamma$ called the power exchange coefficient is defined [22], which is equal to the ratio of transmission power of path 2 at $z=L$ to that of path 1 at $z=0$, as shown in Fig. 10. It is shown that the power exchange coefficient can be expressed as

$$
\gamma=\frac{1}{1+\left(\frac{\Delta \beta}{2 k}\right)^{2}} \sin ^{2}\left(k L \sqrt{1+\left(\frac{\Delta \beta}{2 k}\right)^{2}}\right)
$$

where $\Delta \beta=\omega / c\left(n_{\mathrm{eff} 1}-n_{\mathrm{eff} 2}\right)-2 \pi / \Lambda$ and $n_{\mathrm{eff} 1,2}$ are the effective indexes of the two waveguides, respectively, $k$ is the coupling coefficient, and $\Lambda$ is the grating period. From (14), if $L=\pi /(2 k)$, i.e., one coupler length, at the filter central wavelength $\lambda_{0}=\Lambda\left(n_{\mathrm{eff} 1}-n_{\mathrm{eff} 2}\right)$, a complete power exchange 


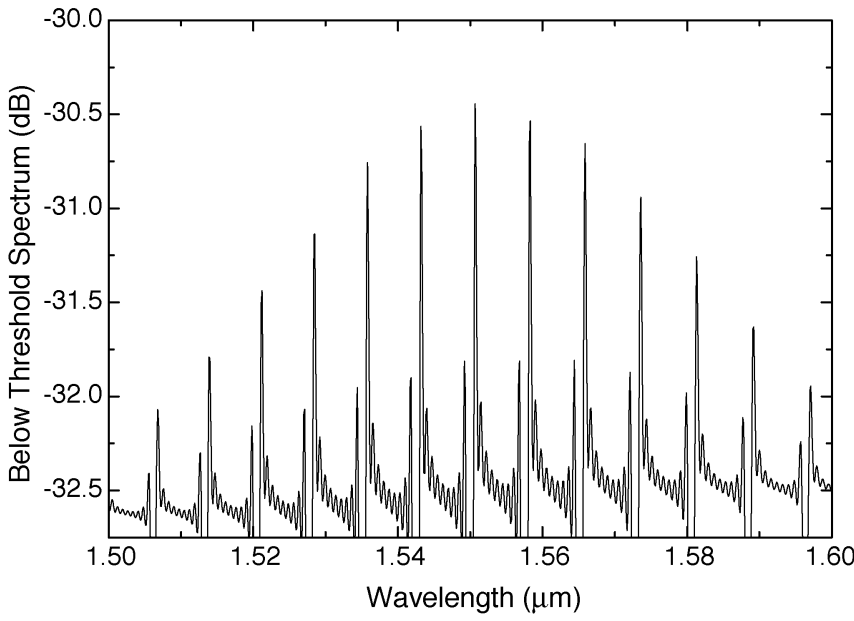

Fig. 9. Below-threshold spectrum of sampled grating gain-coupled DFB laser with both facets AR coated.

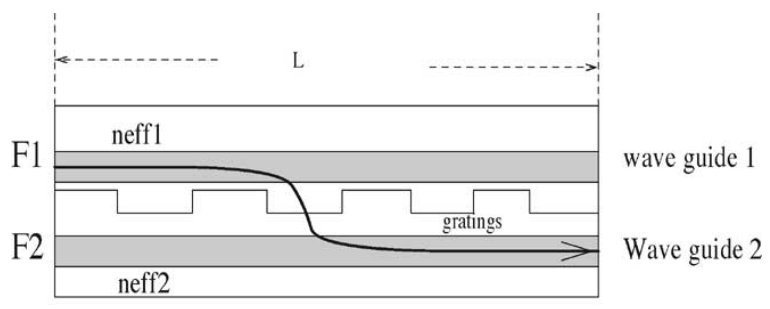

Fig. 10. Grating assisted co-directional coupler filter.

may occur. It can be shown that the bandwidth of filter satisfies the relation

$$
\Delta w \propto \frac{k \lambda_{0}^{2}}{\left(n_{g 1}-n_{g 2}\right)}
$$

and the tuning wavelength range satisfies the relation

$$
\Delta \lambda \propto \frac{\Delta n_{\text {eff } 1}}{\left(n_{g 1}-n_{g 2}\right)} .
$$

Equations (15) and (16) show that tuning range and filter bandwidth both are proportional to the reverse of $n_{g 1}-n_{g 2}$, which is the group index difference coming from the material dispersion. Normally, it can be two to three times larger than the effective index difference [19], [20]. From (15) and (16), it is argued that, in order to extend the tuning range of the filter, a small refractive index difference between the two waveguides is required. On the other hand, to maintain a small bandwidth of the filter, the refractive index difference should be large enough. Therefore, these two requirements must be compromised in the filter design. Finally, to locate the filter central wavelength at the desired wavelength, an appropriate grating period has to be applied. Fig. 11 shows the typical reflectivity and transmission of a co-directional filter while the length $L$ is equal to one filter length, where cleaved facet condition is used at the end $z=L$. We can see that material dispersion leads to great shrinkage of the filter bandwidth.

2) Simulation Results: With the sampled grating DFB in the active section, which provides multimode operation, we cascade a co-directional filter with a cleaved facet at one end of the laser diode as a wavelength selecting and tuning element. The tunable laser structure to be simulated is shown schematically in

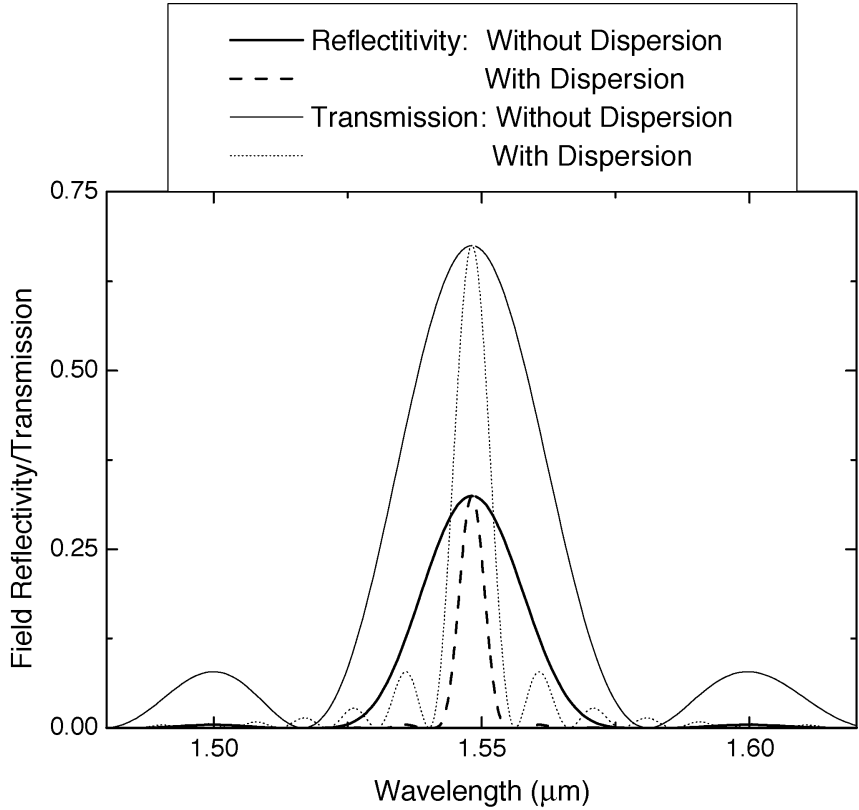

Fig. 11. Reflectivity and transmission of the co-directional filter. Refractive index dispersion plays an important role in the bandwidth.

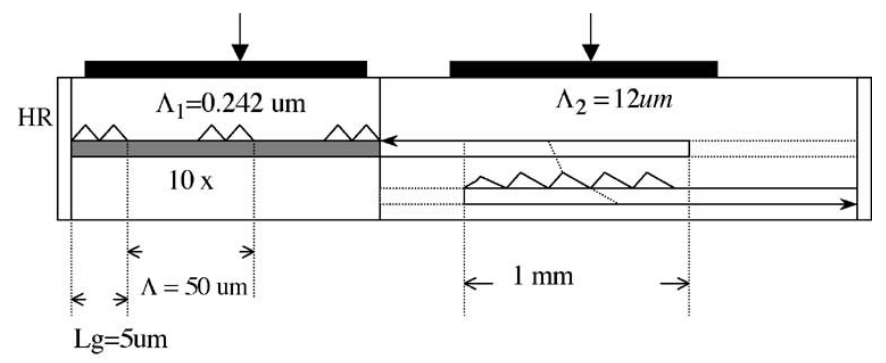

Fig. 12. Sampled grating DFB laser cascaded with a co-directional filter.

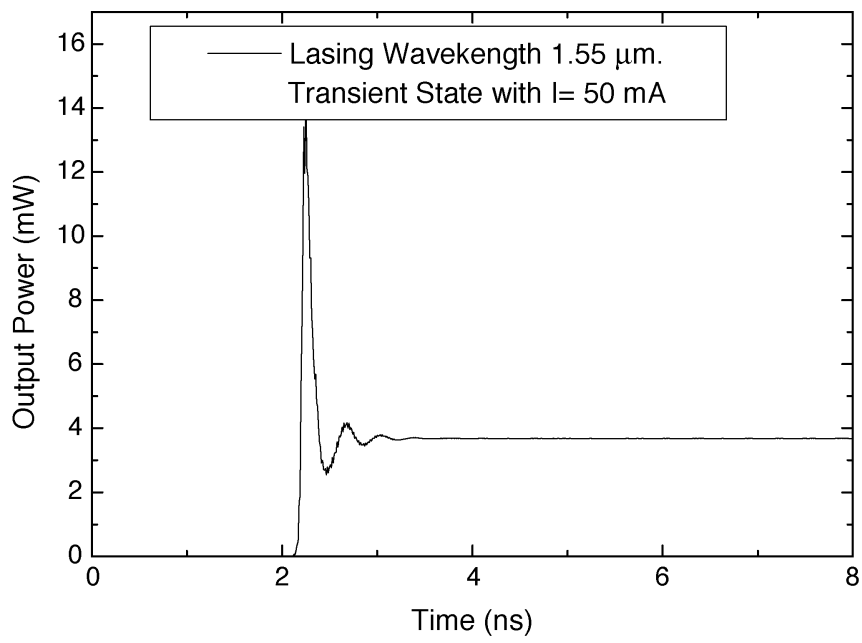

Fig. 13. Transient state when the injected current of active section equals $50 \mathrm{~mA}$ while the tuning current of the co-directional filter is zero.

Fig. 12. For the active section, we choose sampled grating period $\Lambda=50 \mu \mathrm{m}$ and the grating period $\Lambda_{1}=0.242 \mu \mathrm{m}$ with $k L_{g}=0.15,10 \%$ gain coupling and duty cycle $x=L_{g} / \Lambda=$ 0.1 . The total cavity length is equal to $500 \mu \mathrm{m}$. The Bragg wavelength is $1.55 \mu \mathrm{m}$ while there is no current injection. For the co-directional filter, grating period $\Lambda_{2}=12 \mu \mathrm{m}$, the central wavelength of the filter also equal to $1.55 \mu \mathrm{m}$ while there is no 


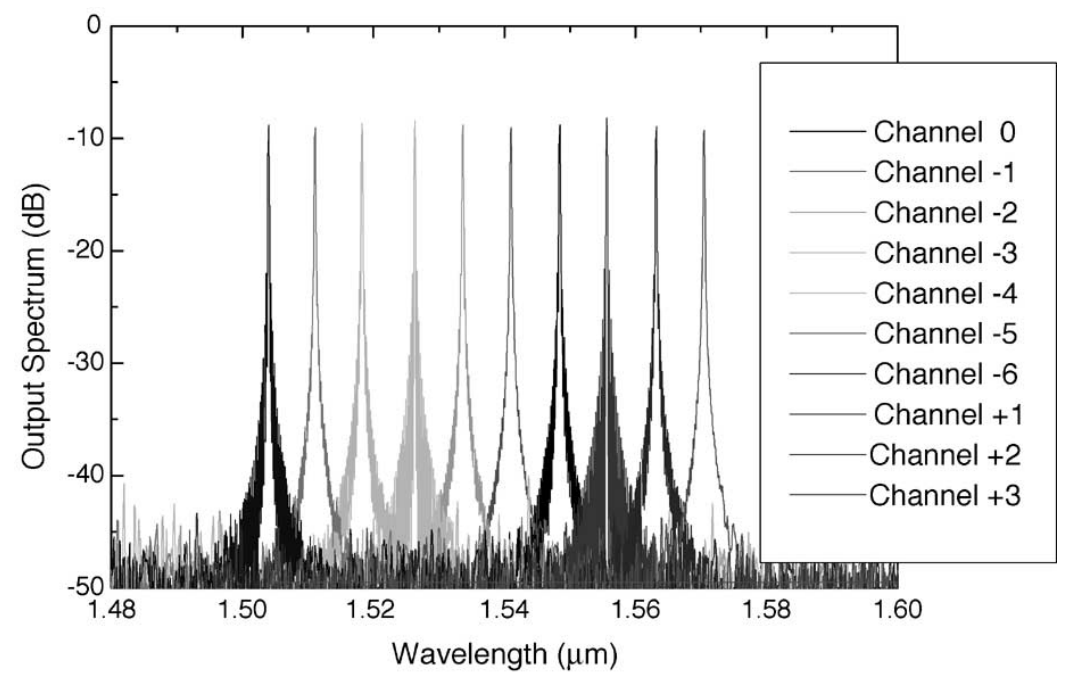

Fig. 14. Turning range and output spectrum of a sampled grating gain-coupled DFB laser with a co-directional filter.

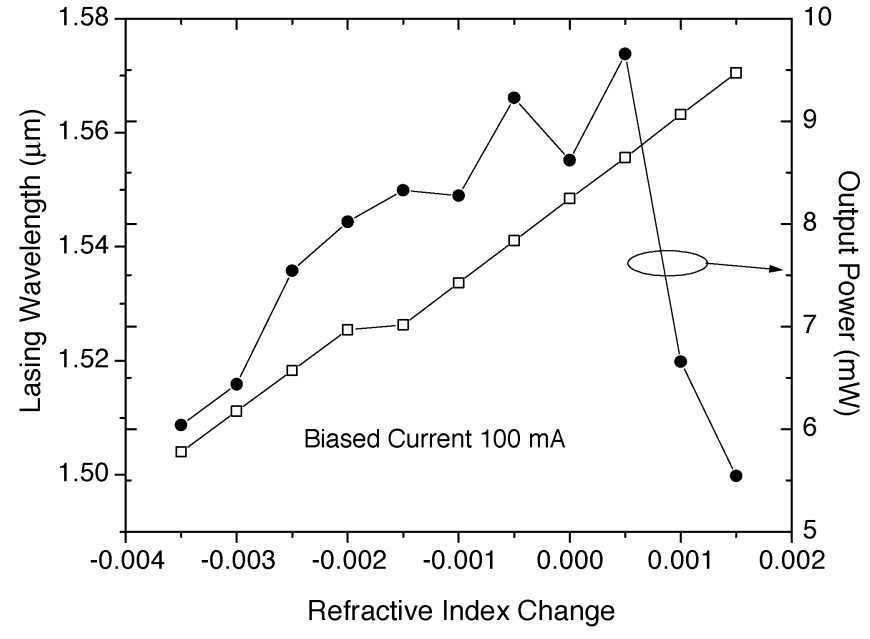

Fig. 15. Operating conditions while the laser is tuned.

tuning current or electric field. The total length of the filter is equal to $1000 \mu \mathrm{m}$, which is one coupling length. In this structure, the adjacent mode spacing of the active sampled grating DFB is around $7.4 \mathrm{~nm}$, while the FWHM of the co-directional filter is less than half the mode spacing. Therefore, by tuning the refractive index in waveguide 1 of the co-directional filter, we can obtain a discrete tunable laser diode.

Using the split-step approach introduced in the previous section, we have simulated the tunable laser diode based on a combination of the sampled grating DFB and a co-directional coupled filter. We should emphasize that, if a full traveling-wave model is used, a specific treatment has to be applied in the co-directional filter section. Particularly in the co-directional filter, the material dispersion and different group velocity between the two waveguides present a great challenge to the full traveling-wave approach [23]. To the best of our knowledge, it is impossible to be realized in the full traveling-wave approach at all. However, in the split-step model, this simulation difficulty can be overcome easily and the approach is also quite general which can be applied to whatever complicated system.

Fig. 13 shows the transient state while the tuning current is equal to zero. While we change the refractive index in waveguide 1 of the co-directional filter, the lasing wavelength will be tuned discretely to display several channels. In Fig. 14, we show the output spectra of these lasing channels in the same graph. As shown in Figs. 14 and 15, this sampled grating DFB wavelength-tunable laser can provide more than $70 \mathrm{~nm}$ tuning range with 10 discrete lasing channels by a refractive index change in the range less than $0.2 \%$.

\section{CONCLUSION}

In this study, we propose a digital filter approach in combination with the traveling-wave model for the simulation of an integrated laser diode and passive components. It is proved that this method is accurate and very efficient in the simulation of the complex passive section cascaded with a laser diode. Using the split-step/digital filter approach, the laser diode is still modeled by the time-domain traveling-wave model or transmission-line model equivalently, but the passive part is simulated by two digital filters butted at the laser diode or the active part end. We present a numerical investigation on a broadly wavelength-tunable laser diode. The integrated photonics device consists of a sampled grating DFB and a co-directional filter, which is difficult or technologically impossible to be solved accurately by the full traveling-wave model. It is concluded that the split-step/digital filter approach is quit successful in the modeling of this kind of complex integrated device. In future work, we will investigate some more complex devices, such as semiconductor optical amplifier (SOA) and AWG integrated multiwavelength sources [24], where the AWG performance could be simulated by the BPM to obtain accurate results [25], while the full traveling-wave model cannot be applied at all for this case. Generally, the method provides a bridge for time-domain modeling (active part) and frequency-domain modeling (passive part) through the digital filters. We believe that, with this new approach, the traveling-wave model will have a broad application in the optical communication system simulation.

\section{ACKNOWLEDGMENT}

The authors would like to thank the reviewers for the valuable comments and advices. 


\section{REFERENCES}

[1] G. Morthier and R. Baets, "Comparison of different DFB laser models within the European COST 240 collaboration," IEE Proc.-Optoelectron., vol. 141, no. 2, pp. 82-88, 1994.

[2] C. F. Tsang, D. D. Marcenac, E. Carroll, and L. M. Zhang, "Comparison between 'power matrix model (PMM)' and 'time domain model (TDM)' in modeling large signal responses of DFB lasers," Proc. Inst. Elect.Eng., pt. J, vol. 141, no. 2, pp. 89-96, 1994.

[3] L. M. Zhang and J. E. Carroll, "Large signal dynamic model of the DFB laser," IEEE J. Quantum Electron, vol. 28, pp. 604-611, Mar. 1992.

[4] X. Li and W.-P. Huang, "Simulation of DFB semiconductor lasers incorporating thermal effects," IEEE J. Quantum Electron., vol. 31, pp. 1846-1855, Oct. 1995

[5] L. M. Zhang, S. F. Yu, M. Nowell, D. D. Marcenac, and J. E. Carroll, "Dynamic analysis of radiation and side mode suppression in second order DFB lasers using time-domain large signal traveling wave model," IEEE J. Quantum Electron., vol. 30, pp. 1389-1395, June 1994.

[6] A. J. Lowery, "New dynamic multimode model for external cavity semiconductor lasers," Proc. Inst. Elect. Eng., pt. J, vol. 136, no. 4, pp. 229-237, 1989.

[7] - "A two-port bilateral model for semiconductor lasers," IEEE J. Quantum Electron., vol. 28, pp. 82-92, Jan. 1992.

[8] — "Computer-aided photonics design," IEEE Spectrum, vol. 34, pp. 26-31, Apr. 1997

[9] W. Li, W.-P. Huang, X. Li, and J. Hong, "Multi-wavelength gain coupled DFB laser cascade: design modeling and simulation," IEEE J. Quantum. Electron, vol. 36, pp. 1110-1116, Oct. 2000.

[10] S. F. Yu and N. Q. Ngo, "Simple model for a distributed feedback laser integrated with a Mach-Zehnder modulator," IEEE J. Quantum Electron., vol. 38, pp. 1062-1075, Aug. 2002.

[11] L. Zhai, A. J. Lowery, and Z. Ahmed, "Locking bandwidth of actively mode-locked semiconductor lasers using fiber-grating external cavities," IEEE J. Quantum Electron., vol. 31, pp. 1998-2005, Nov. 1995.

[12] B. Kim, Y. Chung, and J.-S. Lee, "An efficient split-step time-domain dynamic modeling of DFB/DBR laser diodes," IEEE J. Quantum Electron., vol. 36, pp. 787-794, July 2000.

[13] J. Carroll, J. Whiteaway, and D. Plumb, Distributed Feedback Semiconductor Lasers. $\quad$ London, U.K.: IEE SPIE Opt. Eng. Press, 1998

[14] A. V. Oppenheim and R. W. Schafer, Discrete-Time Signal Processing. Englewood Cliffs, NJ: Pretice-Hall, 1989.

[15] W. H. Press, B. P. Flannery, S. A. Teukolsky, and W. T. Vetterling, Numerical Recipes (Fortran Version). Cambridge, U.K.: Cambridge Univ. Press, 1989.

[16] V. Jayaraman, Z.-M. Chuang, and L. A. Coldren, "Theory, design and performance of extended tuning range semiconductor lasers with sampled gratings," IEEE. J. Quantum. Electron., vol. 29, pp. 1824-1834, June 1993.

[17] H. Kogelnik and C. V. Shank, "Coupled-wave theory of distributed feedback lasers," J. Appl. Phys., vol. 43, pp. 2327-2335, 1978.

[18] J. Hong, K. W. Leong, T. Makino, J. Evans, X. Li, and W. P. Huang, "Impact of random facet phases on modal properties of partly gain-coupled distributed feedback lasers," IEEE J. Select. Topics Quantum Electron., vol. 3, pp. 555-568, Apr. 1997.

[19] T.Toshihiko Makino and J.Jan Glinski, "Transfer matrix analysis of the amplified spontaneous emission of DFB semiconductor laser amplifiers," IEEE J. Quantum Electron., vol. 24, pp. 1507-1518, Aug. 1988.

[20] R. C. Alferness, U. Koren, L. L. Buhl, B. I. Miller, M. G. Young, T. L. Koch, G. Raybon, and A. Burrus, "Broadly tunable InGaAsP/InP laser based on a vertical coupler filter with $57 \mathrm{~nm}$ tuning range," Appl. Phys. Lett., vol. 60, pp. 3209-3211, 1992.

[21] R. C. Alferness, T. L. Koch, L. L. Buhl, F. Storz, F. Heismann, and M. Martyak, "Grating assisted InGaAsP/InP vertical codirectional coupler filter," Appl. Phys. Lett., vol. 55, pp. 2011-2013, 1989.

[22] Z.-M. Chuang and L. A. Coldren, "Design of widely tunable semiconductor lasers using grating -assisted co-directional filters," IEEE J. Quantum. Electron, vol. 29, pp. 1071-1079, Apr. 1993.

[23] B.-S. Kim, J.-K. Y. Chung, and S.-H. Kim, "Time-domain large signal analysis of widely tunable DBR laser diodes with periodically sampled and chirped gratings," IEEE Photon. Technol. Lett, vol. 10, pp. 39-41, Jan. 1998.

[24] M. Zirngibl, C. H. Joyner, C. R. Doerr, L. W. Stulz, and H. M. Presby, "An 18-channel multifrequency laser," IEEE Photon. Technol. Lett., vol. 8, pp. 870-872, July 1996.

[25] M. K. Smit and C. Van Dam, "PHASAR-based WDM-devices: principled, design and applications," IEEE J. Select. Topics Quantum Electron., vol. 2, pp. 236-249, June 1996.
Wei Li (M'99) received the B.S. degree in physics from Jilin University, Changhun, China, in 1989, the M.S. degree in physics from Nankai Institute of Mathematics, Nankai University, Tianjin, China, in 1992, and the Ph.D. degree in electrical engineering from the University of Waterloo, Waterloo, ON, Canada in 2000

In 1999, he joined Apollo Photonics Inc, Burlington, ON, Canada, as a Research Engineer, where he also held an NESERC industry research fellowship. He was in charge of active component simulation software development and device design including strained quantum wells, semiconductor lasers (FP, DFB and DBR), SOAs, electroabsorption modulators, and other integrated active devices. He joined Photonami Inc, Toronto, ON, Canada, in 2001 as a Research Scientist working on the innovative surface emission DFB laser for metropolitan/long-haul optical networks. In 2003, he joined the Department of Chemistry \& Engineering Physics, University of Wisconsin-Platteville, as an Assistant Professor. Now his research interests are the active components for the fiber optical communication system and the quantum effect of nanostructures/devices.

Wei-Ping Huang (M'90-SM'93) received the B.S. degree in electrical engineering from Shandong University, Shandong, China, in 1982 (with provincial and national honors), the M.S. degree from the University of Science and Technology of China, Hefei, in 1984, and the Ph.D. degree from the Massachusetts Institute of Technology (MIT), Cambridge, in 1989, conducting research on fiber and integrated optics, as well as studying international business management at the Sloan School of Management.

He has held a variety of faculty positions in the Electrical and Computer Engineering Department at both the University of Waterloo, Waterloo, ON, Canada, and McMaster University, Hamilton, ON, Canada, including Assistant Professor (1989-1992), Associate Professor with tenure (1992-1996), and Full Professor (1996-present). He has had visiting, adjunct, consulting, and/or managerial positions with several academic and industrial institutions in North America and Asia. He was a Visiting Researcher at Nortel from January to August 1992 and from May to August 1993 and a Visiting Professor at NTT Optoelectronics Lab from September 1995 to August 1996. As the leader of the photonic research group at the University of Waterloo and later at McMaster University, he has carried out a number of major research projects in a wide range of areas of fiber and integrated optics, sponsored by Canadian federal/provincial governments and the private sector. He is internationally known for his contributions and expertise on photonic devices and integrated circuits and has authored and coauthored more than 100 journal papers and 70 conference papers and holds seven U.S. patents.

Dr. Huang was elected to the MIT Electromagnetics Academy and received the Distinguished Leadership Award from the American Biographical Institute in 1996. He was elected as a Cheung Kong Scholar by the Ministry of Education, R.O.C., and the Li Ka Shing Foundation, Hong Kong, in 2000. He was the Founding President of the Shandong University Oversea Alumni Association and is the Founding President of the Jinan Overseas Chinese Scholars and Professional Association. He is a Member of the Optical Society of America and the International Society for Optical Engineers (SPIE).

Xun Li (M'93) received the B.S. degree from Shandong University, Jinan, China, in 1982, the M.S. degree from the Research Institute of Posts Telecommunications, Wuhan, China, in 1984, and the Ph.D. degree from Northern Jiaotong University, Beijing, China, in 1988.

He joined the faculty of the Lightwave Technology Institute, Northern Jiaotong University, in 1988, where he was a Professor. He was a Research Assistant Professor with the Department of Electrical and Computer Engineering, University of Waterloo, Waterloo, ON, Canada, from 1993 to 1999 . He joined the faculty of Engineering at McMaster University, Hamilton, ON, Canada, in 1999, where he is now an Associate Professor. He is mainly working in the area of optical fiber communication systems and quantum electronics, with emphasis on computer-aided modeling, simulation, and design of optoelectronic devices such as semiconductor lasers and amplifiers and their applications on optical fiber communication systems and networks 